\title{
Santa Maria di Agnano (Ostuni, Puglia)
}

Donato Coppola, Nicola De Pinto, Michele Pellegrino, Henry Baills, Martine Dewailly, Jacopo Russo et Jessica Bartolomeo

\section{OpenEdition \\ Journals}

\section{Édition électronique}

URL : http://journals.openedition.org/cefr/1482

DOI : $10.4000 /$ cefr. 1482

ISSN : 2282-5703

Éditeur

École française de Rome

\section{Référence électronique}

Donato Coppola, Nicola De Pinto, Michele Pellegrino, Henry Baills, Martine Dewailly, Jacopo Russo et Jessica Bartolomeo, "Santa Maria di Agnano (Ostuni, Puglia)», Chronique des activités archéologiques de l'École française de Rome [En ligne], Italie du Sud, mis en ligne le 22 février 2016, consulté le 19 avril 2019. URL : http://journals.openedition.org/cefr/1482 ; DOI : 10.4000/cefr.1482

Ce document a été généré automatiquement le 19 avril 2019.

(c) École française de Rome 


\section{Santa Maria di Agnano (Ostuni, Puglia)}

Donato Coppola, Nicola De Pinto, Michele Pellegrino, Henry Baills, Martine Dewailly, Jacopo Russo et Jessica Bartolomeo

\section{La frequentazione neolitica esterna e le testimonianze rituali ellenistiche nell'area $\mathrm{H}-\mathrm{I}-\mathrm{P}-\mathrm{Q}$ (Donato Coppola, Nicola de Pinto, Michele Pellegrino)}

\section{La frequentazione neolitica esterna}

Le indagini archeologiche del 2015 a Santa Maria di Agnano ad Ostuni hanno interessato le aree di scavo già esplorate nel 2011 : l'estensione dello scavo del muro di recinzione e l'esplorazione dei livelli olocenici e pleistocenici sottostanti la parete rocciosa del riparo nell'Area H-I-P-Q; i settori identificati come "Scavo esterno", riferibile alle stratificazioni paleo-epipaleolitiche; le Aree L-M, pertinenti alla terrazza inferiore dove sono attestati strati d'epoca ellenistica (fig. 1). 


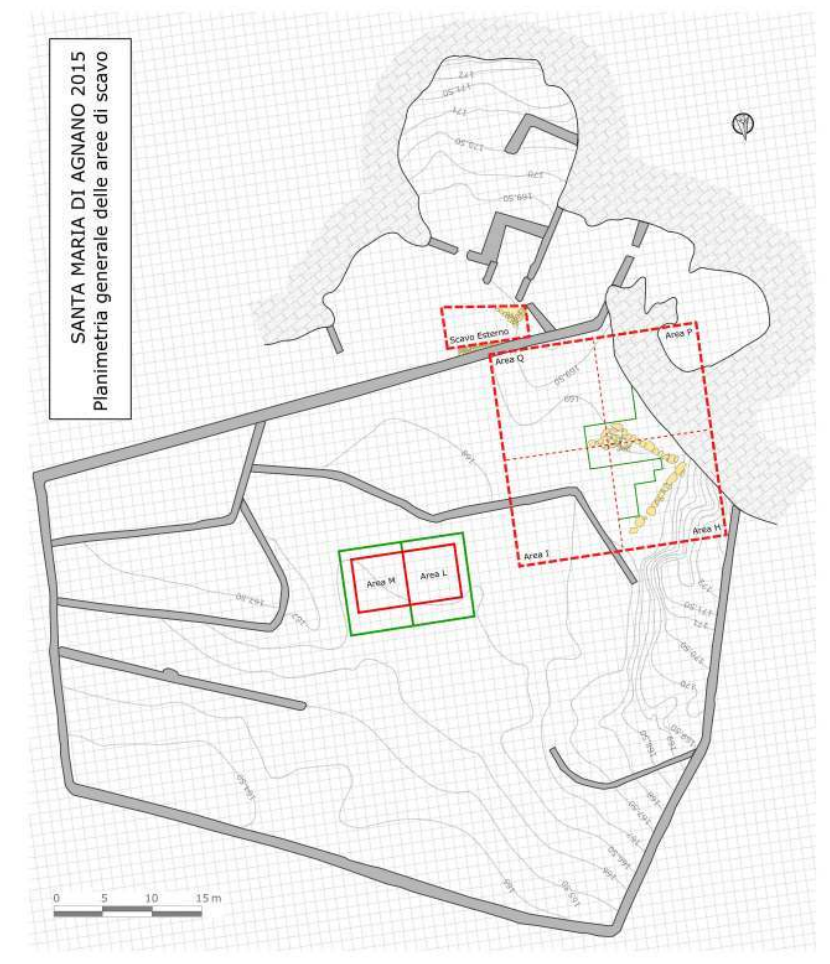

MiCHELE PELlegRino.

Nell'area H-I-P-Q corrispondente al grande riparo calcareo di Agnano che piomba con una ripida parete verticale ed alla cui base doveva aprirsi la parte frontale interrata della retrostante cavità occidentale, un considerevole accumulo di terreno rossastro ci ricollega allo scavo del 2011. La ripulitura nell'ambito dei quadrati H e P aveva evidenziato come la posa dei massi calcarei della fondazione del peribolo ovest fosse avvenuta con un incasso dei blocchi in un taglio operato in strati più antichi, caratterizzati per lo più da ceramiche neolitiche e industrie litiche paleo-epipaleolitiche $\mathrm{e}$ faune pleistoceniche, contenute in una matrice di terreno rossastro.

Lo sfoglio per décapage dei livelli olocenici ha identificato tre aree caratterizzate da una forte presenza di terra combusta mista a cenere che indicherebbe una frequentazione neolitica con l'installazione di alcuni focolari : il Focolare 1, nei quadrati Ha4, Pl4 (fig. 2), si presenta superiormente (1a) con un accumulo esteso di terreno poco compatto, di colore bruno rossastro con matrice terroso-argillosa e forti tracce di combustione, che diventa nell'area sottostante (1b) nerastro e ricco di materiale carbonioso. Nella parte basale sono presenti lastrine calcaree calcinate (1c) ed alcuni frammenti riconducibili ad un vaso pithoide ad impasto bruno con fondo a tacco rilevati anche nelle aree esterne al focolare (fig. 2). La presenza abbondante di intonaco e concotto con resti di piccole impronte di rami o canne e l'intensità della combustione fanno pensare ad un contesto rituale che potrà essere meglio definito con le analisi paleobotaniche dei sedimenti prelevati ma che ci riporta per analogia tipologica ai materiali del sito neolitico di Torre Canne ${ }^{1}$. 
Figure 2 - Rilievo area H-I-P-Q con dettaglio della quadrettatura interna (2015).

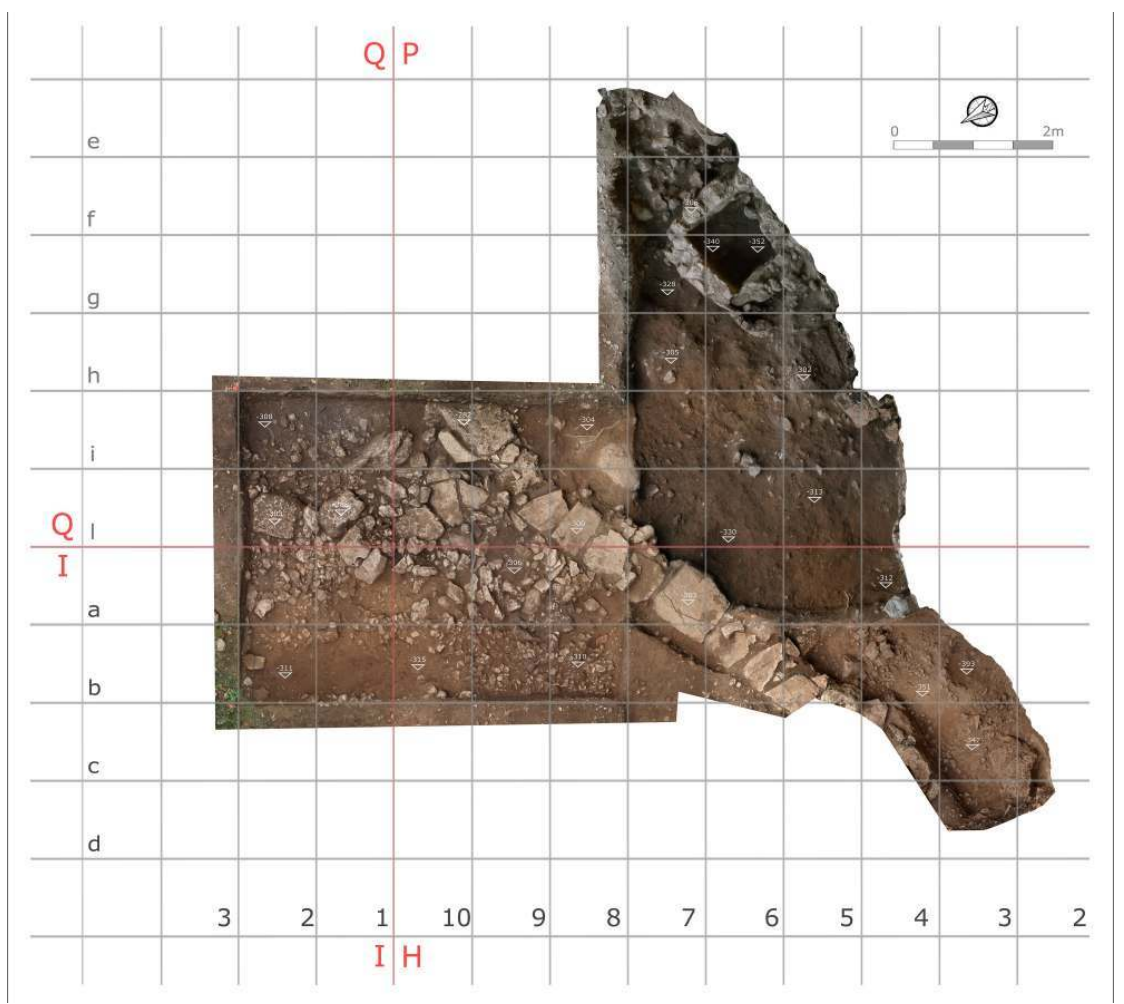

MICHELE PELLEgRino.

4 Nei quadrati Pi-l8, si evidenzia il Focolare 2 formato da due livelli : nel livello superiore si registra la presenza di materiale ceramico estremamente frammentario di età Neolitica (ceramica impressa, graffita e scarsamente dipinta a fasce-chiazze rossastre) e, limitatamente ai quadrati $\mathrm{Ph}-\mathrm{i}$, un secondo livello in cui sono stati rinvenuti resti faunistici e industria litica. Altri residui di chiazze (Focolari 3 e 4) sono di difficile attribuzione, poiché è evidente che queste fasi del Neolitico antico all'esterno si sviluppano su preesistenze epipaleolitiche, confermando quanto già intravisto nel saggio del 1996 dove un focolare a catino con pietre di cottura della stessa fase si sovrapponeva ad un livello del tardo-epigravettiano con presenza del circolo rituale $2^{2}$. 
Figure 3 - Area di combustione neolitica.

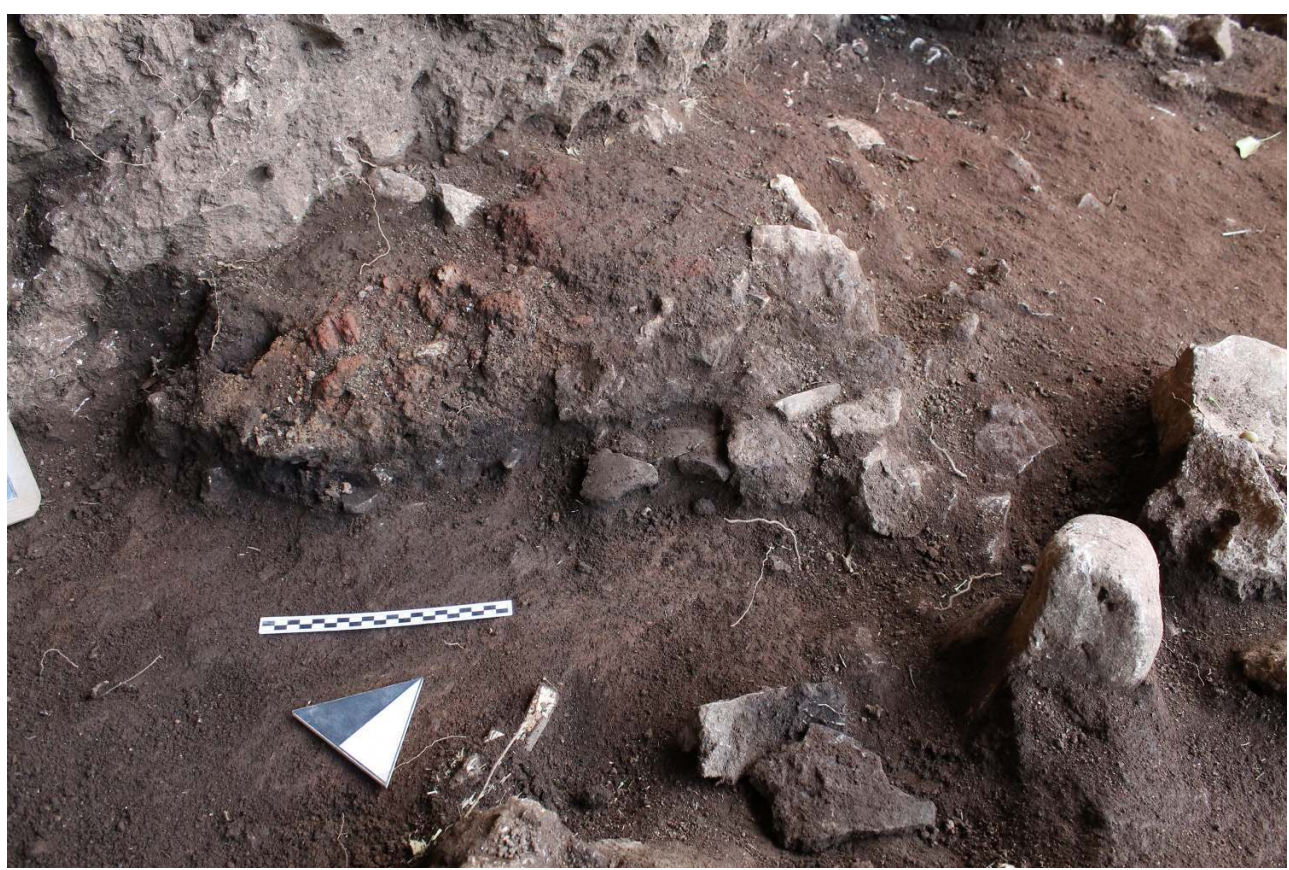

Donato Coppola

L'aver intravisto l'affioramento di uno strato già tendenzialmente romanelliano con micrograttatoi circolari, linguette di Spondylus anche con decoro a tratti incisi, numerosi frammenti ossei con motivi incisi a tratteggio o a «scala ", associati ad una fauna in fase di studio, già tardo pleistocenica-mesolitica con abbondanza evidente di cervo, cinghiale, tartarughe e piccoli selvatici, ci ha costretti a sospendere lo scavo in questa fase per riprenderlo sistematicamente nel 2016 con l'esplorazione di questo deposito preolocenico di eccezionale interesse scientifico, probabilmente rapportabile alla datazione radiometrica calibrata LTL2514A ( 9700 - 9290 cal. B.C.) ottenuta nell'area dello Scavo esterno, in $d 36$, livello 2.

\section{Le testimonianze rituali ellenistiche nell'Area $\mathrm{H}-\mathrm{I}-\mathrm{P}-\mathrm{Q}$}

6 Ad ovest, sulla terrazza superiore, si è proseguito lo scavo del muro di peribolo che, con orientamento nord-sud, si sviluppava per una lunghezza di circa 9,30 m, composto da blocchi calcarei, alcuni molto ben squadrati (a nord), altri grossolanamente sbozzati. A sud il muro, dopo l'ultimo masso di chiusura, fa angolo a $90^{\circ}$ in direzione est-ovest e presenta un abbassamento strutturale dovuto forse ad una piccola soglia di accesso. Il muro di peribolo è stato evidenziato ancora per circa $8 \mathrm{~m}$ : è composto sempre da lastroni squadrati e spessi, di sfaldamento calcareo, disposti come delimitazione di un'area interna utilizzata per il culto con numerose testimonianze di un'intensa attività rituale tra IV e III sec. a. C. L'interruzione in direzione ovest definisce pertanto non la base di un'eventuale fondazione di un muro perimetrale ma una recinzione alle aree di culto interne, in parte esplorate (fig. 4). 
Figure 4 - Rilievo delle strutture nelle aere di scavo H-I-P-Q.

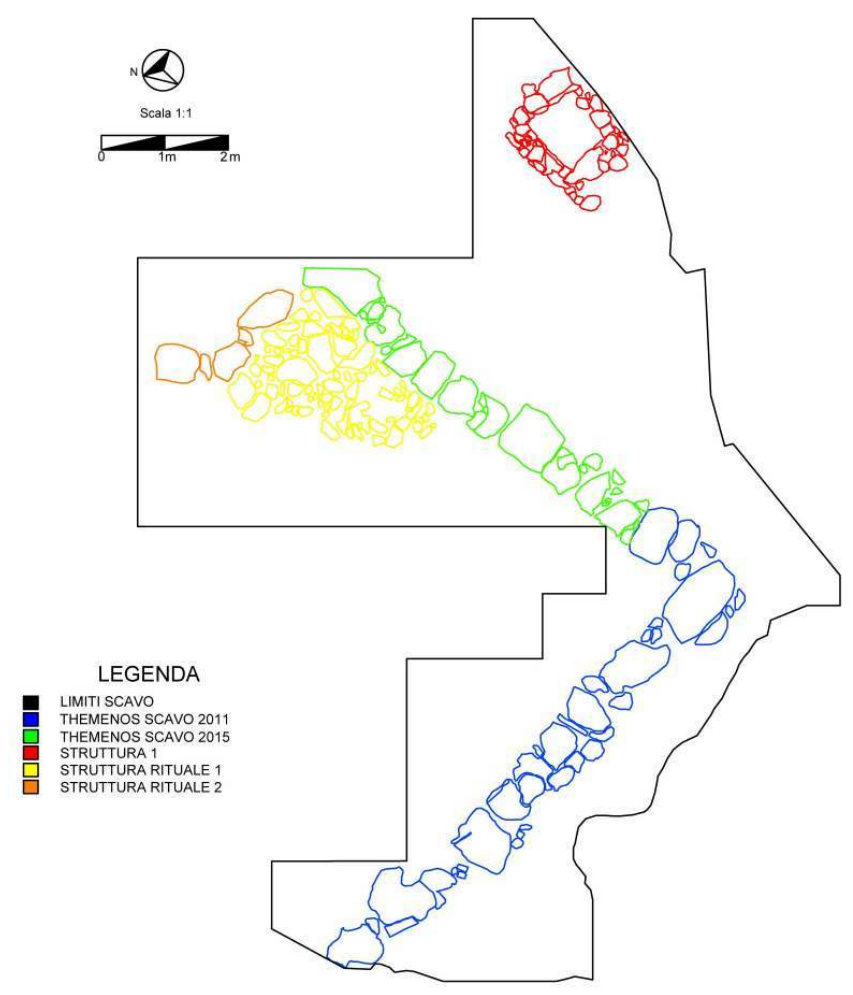

Nicola De Pinto.

\section{Area Rituale 1, livelli 1 e 2}

7 La struttura più o meno quadrata si addossa al muro del peribolo ed è foderata da blocchi calcarei appiattiti e lastre, per una larghezza di circa 2 × $2 \mathrm{~m}$. I livelli 1 e 2 si presentano con un accumulo terroso ricco di elementi carboniosi-cinerei e pietre lavorate di medio modulo (addossate alle pareti della struttura e nella sua parte centrale) collocate nei quadrati Ha9-Ha10, Pi9-Pi10 con pavimentazione di lastre calcaree, estesa nei quadrati Ha8 -Ha9 e nei quadrati Pl8-Pl9. Lo scavo evidenzia la presenza di due altari: uno in calcarenite, appiattito e con bordo parzialmente rilevato, in situ e addossato sul perimetro orientale, l'altro, un masso calcareo trapezoidale adagiato per il lato lungo sul fondo ben rifinito e con forti tracce di combustione ${ }^{3}$ (fig. 5). 
Figure 5 - Area Rituale 1, livello 1.

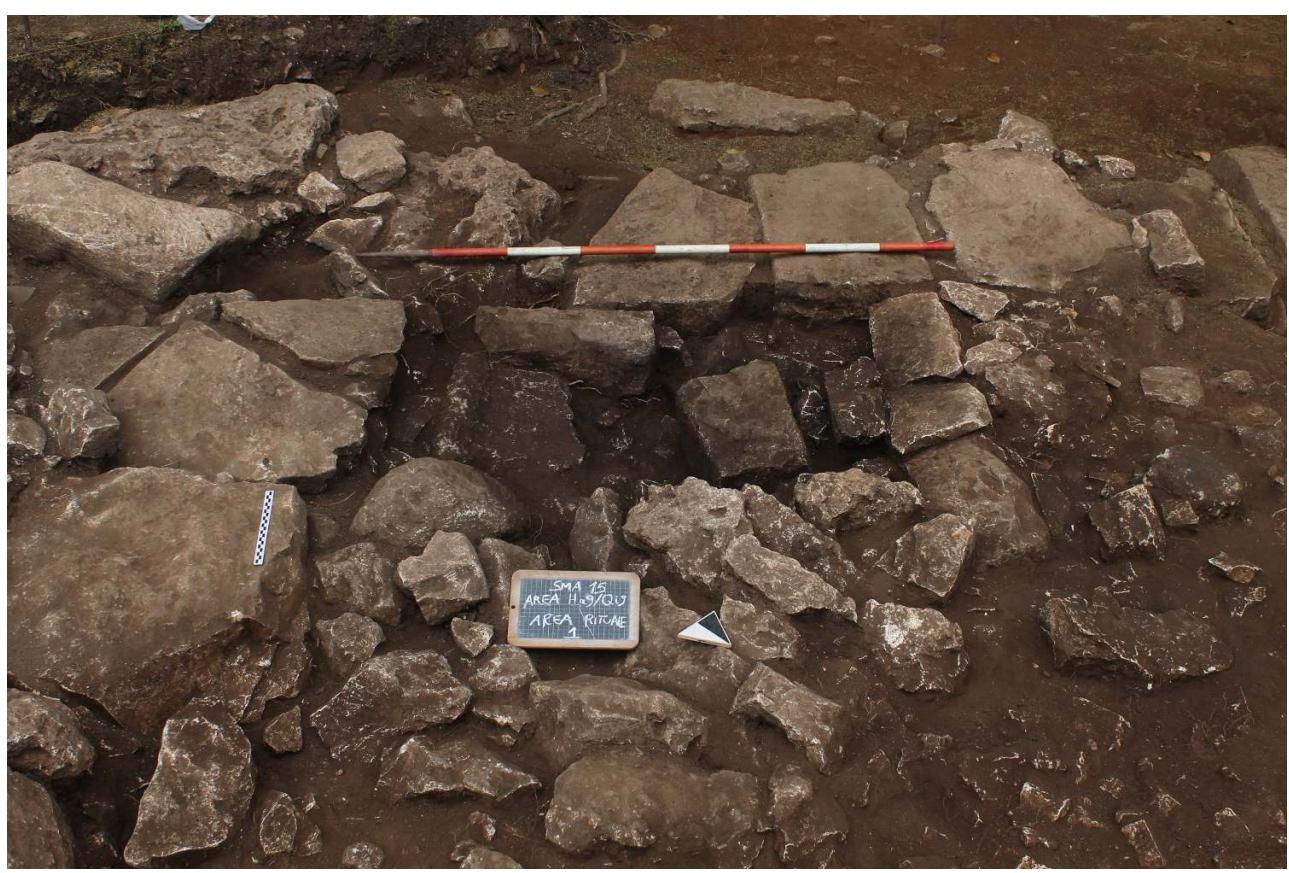

Donato Coppola

Tra i frammenti rinvenuti all'interno, testimoni di un utilizzo già nel corso del IV sec. a.C., si segnala una protome di tipo demetriaco con capelli fluenti ondulati intorno al capo, sormontati da un fregio e con alto pòlos (fig. 6).

Figure 6 - Protome area rituale 1.

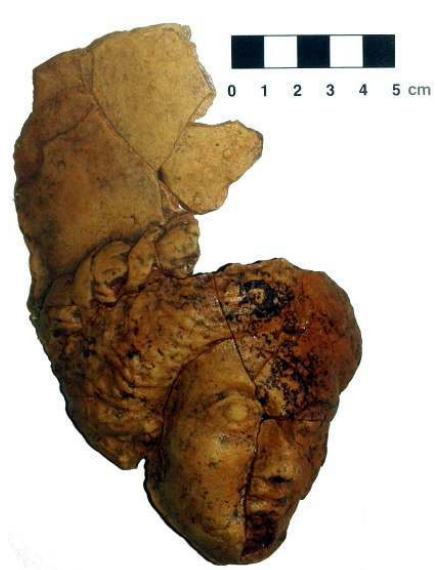

Donato Coppola

Nell'angolo nord-ovest, è stata rinvenuta un'anfora globosa in miniatura simile ad un reperto già segnalato nella raccolta archeologica del Capitolo Cattedrale di Ostuni e proveniente dalla necropoli messapica della Rosara, in contesti della fine del IV-inizi III sec. a.C.4. Le attestazioni di un uso protratto dell'area sacra sono documentate dalla presenza di diversi riempimenti di tegole che, insieme a del pietrame, coprono tutta l'area votiva dell'area Ha8. 


\section{Area rituale 2, livelli 1-5}

8 Ad est dell'area rituale 1, si addossano altri blocchi calcarei che tendono a definire una parziale recinzione curvilinea all'interno della quale vi sono tracce consistenti di attività rituali, solo in parte esplorate. Il livello 2 è uno strato di accumulo abbastanza compatto di colore bruno-nerastro con matrice terrosa mista a tracce di terra argillosa con forti tracce di combustione, esteso nei quadrati Qi1-Qi2, Ql1-Ql2 e Pi10-Pl10. I blocchi calcarei di medio-grande modulo, che si addossano definendo il margine occidentale dell'area, poggiano sull'interfaccia inferiore del livello 2 dell'Area rituale 2 : nel riempimento sono stati rinvenuti frammenti ceramici parzialmente ricomponibili tra le US 61-62. Si segnala in particolare una grande hydria decorata a fasce : il dorso dell'ansa a nastro è decorato con linee disposte a "croce di Sant'Andrea » identificate come simbolo demetriaco in numerose iscrizioni messapiche riferibili al III sec. a. C. ${ }^{5}$. Il sottostante livello 3 è uno strato di accumulo abbastanza compatto di colore bruno-nerastro, composto da una matrice terrosa e caratterizzato da una cospicua presenza di tegole; spicca il rinvenimento di una punta lanceolata in ferro (fig. 7), lunga $18 \mathrm{~cm}$, posta verticalmente nell'interfaccia del successivo livello 4 .

Figure 7 - Area rituale 2 livello 3 base.

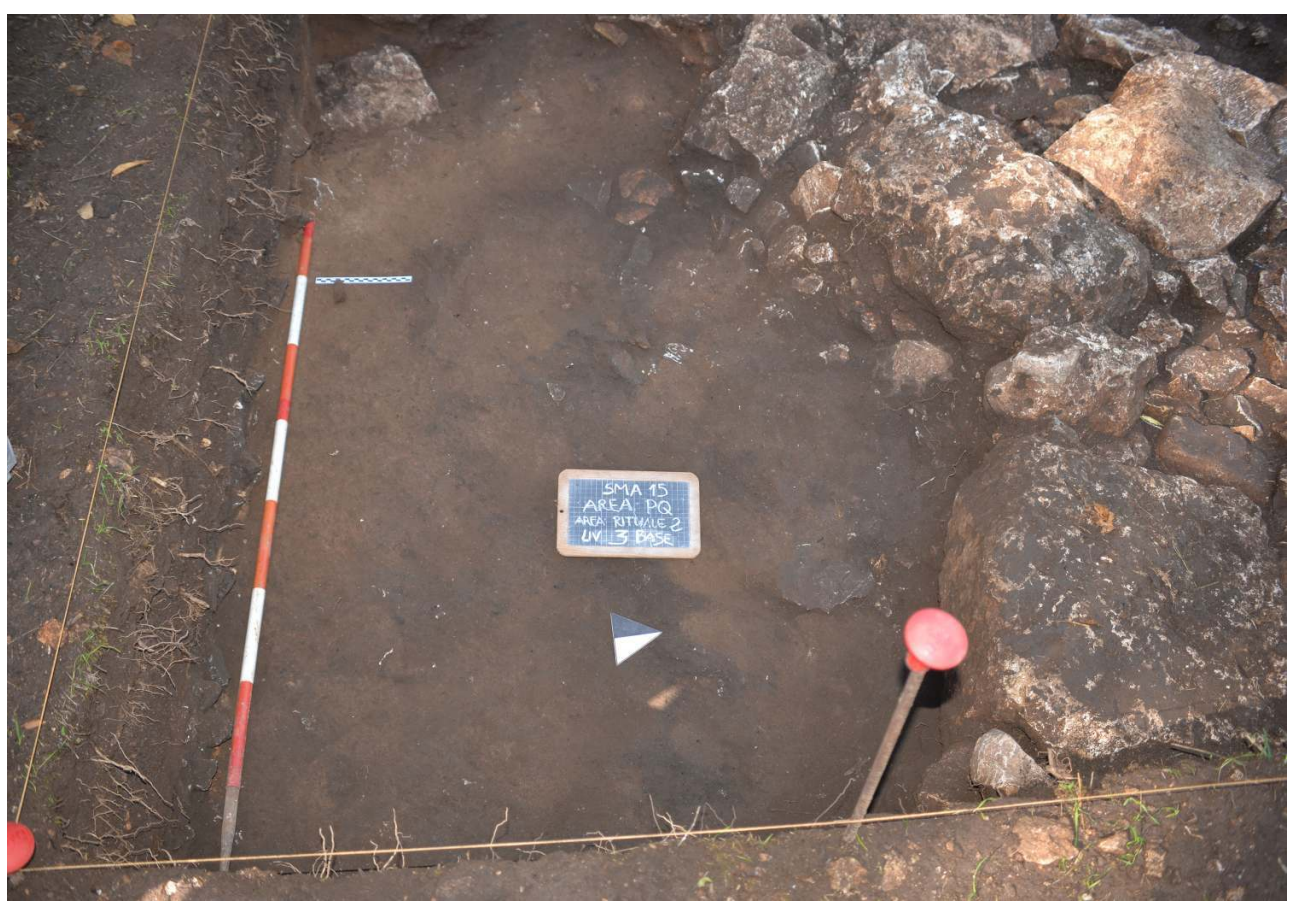

Donato Coppola

9 Quest'ultimo è un deposito naturale compatto di colore bruno-marrone a matrice sabbiosa, sovrastante il deposito terroso-sabbioso con scarsa presenza di frammenti ceramici del Neolitico e dell'età del Bronzo. 


\section{SMA - Esterno (Henry Baills)}

Avec l'accord de Donato Coppola, la fouille de l'aire SMA-Esterno a été reprise au cours du mois d'octobre 2015. Cette aire couvre une surface de $21 \mathrm{~m}^{2}$ (fig. 1 et $8 \mathrm{a}$ ) située immédiatement à l'avant de l'aplomb rocheux du porche actuel. La campagne de fouille a concerné une superficie, volontairement restreinte, de $3 \mathrm{~m}^{2}$ correspondante aux carrés c1, c2 et d3 (fig. 8b) et avait l'objectif spécifique de tenter de répondre à une double problématique qui se posait suite à la fouille de $2011^{6}$ :

- Quel crédit accorder à l'hypothèse d'une présence aurignacienne avancée sur la base d'artefacts de type aurignacoïde découverts en 2011 ?

- Comment les occupations du Gravettien (US 9-US 8) et les blocs liés à l'effondrement du porche (US 5A) se positionnement-ils respectivement (fig. 8c)?

Figure 8 - Plans et stratigraphie.
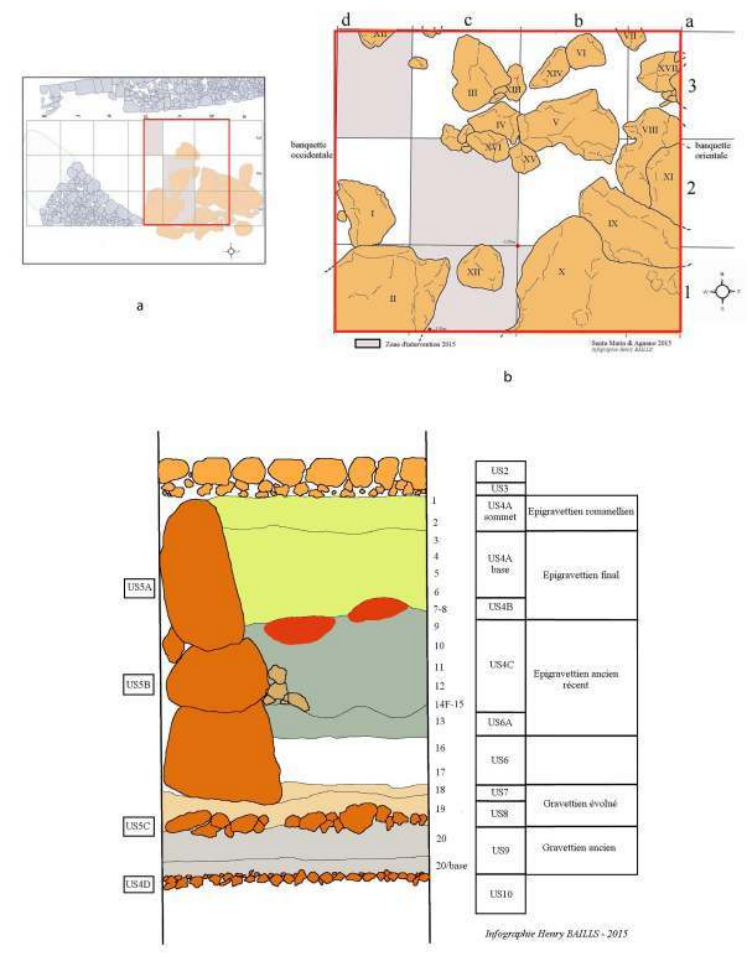

Henry Baills.

11 En octobre 2015, ont été concernées seules les unités stratigraphiques US 9 et US 8 qui se présentent comme un sédiment sombre à matrice argileuse qui doivent leur couleur noirâtre à la dégradation de matières organiques.

Dans la zone c1 et c2, l'US 9 a été subdivisée en deux niveaux, 20/base (ép. $20 \mathrm{~cm}$ ) et 20 (ép. $7 \mathrm{~cm}$ ), l'US 8 comprend également deux niveaux, 19 (ép. $5 \mathrm{~cm}$ ) et $17(8 \mathrm{~cm})$. En zone d3, seul le niveau 20/base (ép. $7 \mathrm{~cm}$ ) de l'US 9 a été identifié.

Nos observations de terrain permettent de penser que les niveaux 20/base et 20 de l'US 9 sont sous-jacentes à l'amas de blocs (III, IV, V, X, XIII, XV, XVI) de moyen module dans la 
partie nord de la fouille tandis que les niveaux 19 et 17 de l'US 8 sont des formations de faible puissance directement positionnées sous les grands blocs (II, IX et X) dans la partie sud de la fouille.

L'industrie lithique de l'US 9 est réalisée sur des supports morphologiquement variés. On remarque, en effet, à la fois l'utilisation de supports lamino-lamellaires de bonne facture issus des phases de plein débitage conjointement avec celle d'éclats épais résultant des opérations initiales de mis en forme volumétrique du nucléus. Certains outils gardent encore sur un de leurs pans des plages résiduelles du cortex original (fig. $9 \mathrm{n}^{\circ} 2,4,7$ ), preuve que les tailleurs ont géré avec parcimonie la matière première siliceuse couleur miel de bonne qualité provenant du Gargano à $200 \mathrm{~km}$.

- Les lames sont les supports privilégiés des burins sur cassure (fig. $9, \mathrm{n}^{\circ} 4$ ), sur troncature (fig. $9 \mathrm{n}^{\circ} 1$ ), ou mixtes (fig. $9 \mathrm{n}^{\circ} 8$ ).

- Non figurées ici, les pointes à dos de type Gravette sont portées par des lamelles de faibles dimensions. Palma di Cesnola avait déjà observé ce phénomène de microlithisation des pointes à dos dans les strates 23 et 22 du Gravettien ancien de la grotte Paglicci (Foggia).

- Les éclats épais ont été choisis pour la réalisation des grattoirs carénés (fig. 9 n² 2, 3, 7). La pièce $n^{\circ} 3$, identifiée comme un grattoir caréné brisé, possède un faux air de pièce de La Bertonne telle qu'on l'a reconnue dans le Sud-Ouest de la France. À y regarder de plus près elle ne réunit pourtant pas tous les critères qui caractérisent formellement cette pièce. Il est vrai qu'une pièce de La Bertonne s'accorderait mal avec l'ambiance gravettienne des niveaux de l'US 9 !

Figure 9 - Industrie lithique.
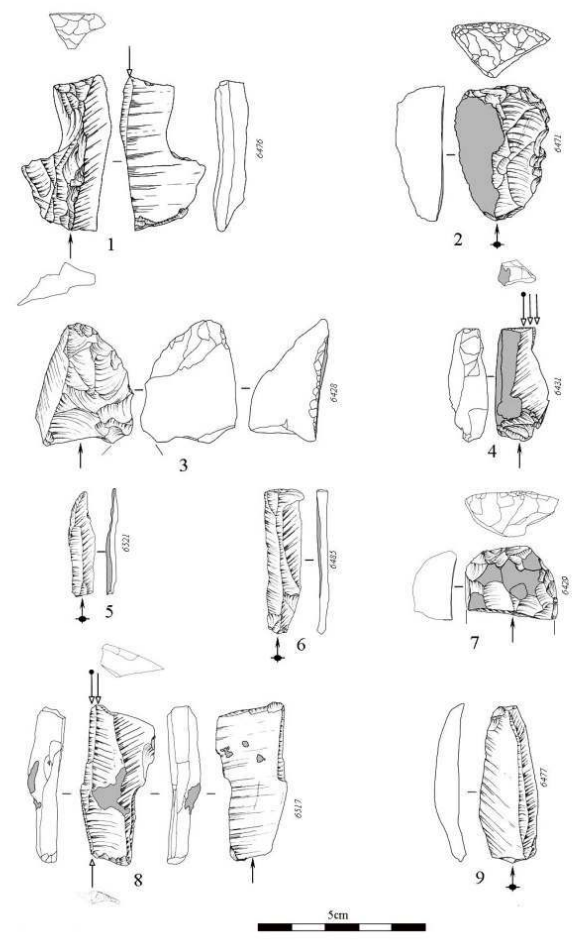

Henry Baills. 
L'industrie recueillie au cours de la fouille 2015 constitue à ce jour la base de la séquence gravettienne du site de SMA-Esterno. Elle correspond à une phase ancienne du Gravettien de la Pouille. Ce moment initial du Gravettien est bien connu dans les strates 23-22 de la grotte Paglicci. On y retrouve la présence des grattoirs et burins carénés associée à des microgravettes; tableau identique à celui de l'US 9 de SMA-Esterno. Dans l'attente d'une datation ${ }^{14} \mathrm{C}$ sur des charbons prélevés directement de la strate archéologique dans les carrés $\mathrm{d} 1$ et $\mathrm{d} 2$, on peut penser que celle obtenue pour la strate 23 de Paglicci de $28100 \pm$ 400 B.P. 7 pourrait s'appliquer aux niveaux 20/base-20 de l'US 9 de SMA-Esterno.

L'US 9 a également livré des restes fauniques en bon état de conservation. Quelques spécimens ont été déterminés par Pierre Magniez ${ }^{8}$. Il s'agit d'ossements de Bos primigenius (fig. 10 a) et d'Equus ferus (fig. 10 b et c). Cette association est fréquente dans le Pléistocène supérieur de l'Italie.

Figure 10 - Restes fauniques.
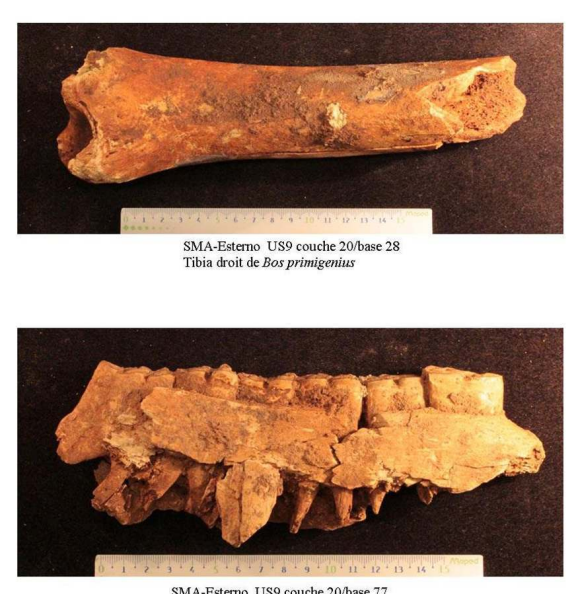

SMA-Esterno US9 couche 20, base 77
Mandibule droite d'Rquus ferus avec $\mathrm{P} 2$ a a3

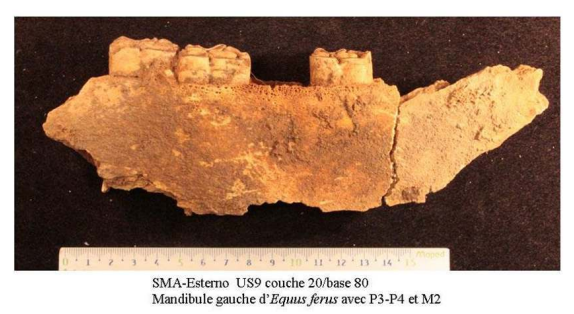

Henry Baills, Pierre Magniez.

17 En fin de fouille, immédiatement sur l'US 9, niveau 20/base, nous avons remarqué dans les carrés $\mathrm{c} 1$, c2 et d3 la présence d'une formation subhorizontale associant des blocs de petites dimensions à une argile rougeâtre que nous avons appelée US 10.

Il ressort de ces observations stratigraphiques que la dynamique de l'effondrement du porche doit être envisagée suivant un mode complexe. Le recul de l'auvent a alterné des moments de simple desquamation provoquant le décrochement d'éléments rocheux de faible taille et des temps d'effondrements plus importants. La forte hétérogénéité des modules des blocs et leur répartition spatiale sur l'aire fouillée va dans le sens d'une série de phases plutôt que d'un événement unique. 
19 Si l'on tente de corréler le scénario des effondrements avec la succession des chronocultures préhistoriques il ressort que l'occupation du Gravettien ancien, qui a fait l'objet de la fouille 2015, se positionne sous l'amas des blocs nord (III, IV, V, X, XIII, XV, XVI) de module moyen à faible. Elle semble s'être déroulée durant un moment de faible effondrement du porche et peut-être de rigueur climatique moindre. On peut avancer l'IS 3 ou IS 4 de Dansgaard-Oeshger. Une datation ${ }^{14} \mathrm{C}$ attendue devrait amener une réponse à cette hypothèse.

L'occupation du Gravettien évolué, fouillée en 2011, est antérieure à un écroulement majeur correspondant aux gros blocs sud (II, IX et X). Cette péjoration climatique doit correspondre à l'événement de Heinrich 2, entre 18000 et 16000 B.P. ce qui donnerait un certain crédit à la date ${ }^{14} \mathrm{C}$ obtenue pour l'US 8 niveau 17 de $23945 \pm 110$ B.P.

21 La perspective immédiate pour 2016 visera à poursuivre l'exploration extensive de l'US 9, niveaux 20/base et 20 , de façon à documenter plus largement la série lithique trouvée en 2015 dans cette formation. Le Gravettien ancien de SMA-Esterno viendrait alors compléter nos connaissances sur ce moment initial du Gravettien reconnu seulement dans les strates 23-22 de la grotte Paglicci.

D'autre part, il serait intéressant de vérifier que l'US 10 dégagée en fond de fouille en 2015, perçue comme archéologiquement stérile, l'est réellement, et enfin de tester, par un sondage profond, la validité d'une présence aurignacienne sur le site.

\section{Area M-L \\ (Martine Dewailly, Jacopo Russo, Jessica Bartolomeo)}

23 L'esplorazione archeologica sulla terrazza inferiore, antistante alla grotta, è stata ripresa nei mesi di giugno e settembre 2015, dopo tre anni d'interruzione ${ }^{9}$. L'area, allargata grazie all'ausilio di un escavatore, si estende su $96 \mathrm{~m}^{2}$ ed è divisa in due quadrati $\mathrm{M} \mathrm{e} \mathrm{L}$, di $6 \mathrm{x}$ 8 metri ciascuno (fig. 1).

Con il passaggio dell'escavatore si è proceduto alla rimozione degli strati agricoli ${ }^{10}$. Al fine di ritrovare uno strato archeologico chiaro, il livello lasciato dall'escavatore, identificato come US 31, è stato scavato ed ha restituito del materiale ceramico antico e rinascimentale. Si tratta di uno strato agricolo che fa da contatto con il primo strato archeologico US 21. Inoltre si caratterizza per la presenza delle prime pietre del crollo localizzato nell'area M nel 2011; la pulizia di quest'ultimo ha portato alla scoperta di un'antefissa frammentaria. L'antefissa (fig. 11), di forma ellittica, conserva parte della capigliatura, la fronte, gli occhi, il naso ${ }^{11}$, e raffigura il volto di Medusa ${ }^{12}$; inoltre conserva parte del $\operatorname{coppo}^{13}$. La fattura dell'antefissa di Agnano, che presenta una capigliatura diversa da quella di Taranto, è di minore qualità, il che suggerisce che deriva da un'altra matrice ed è di una generazione successiva. Si propone dunque una datazione compresa tra il secondo e il terzo quarto del IV secolo ${ }^{14}$. 
Figure 11 - Antefissa con volto di Medusa.
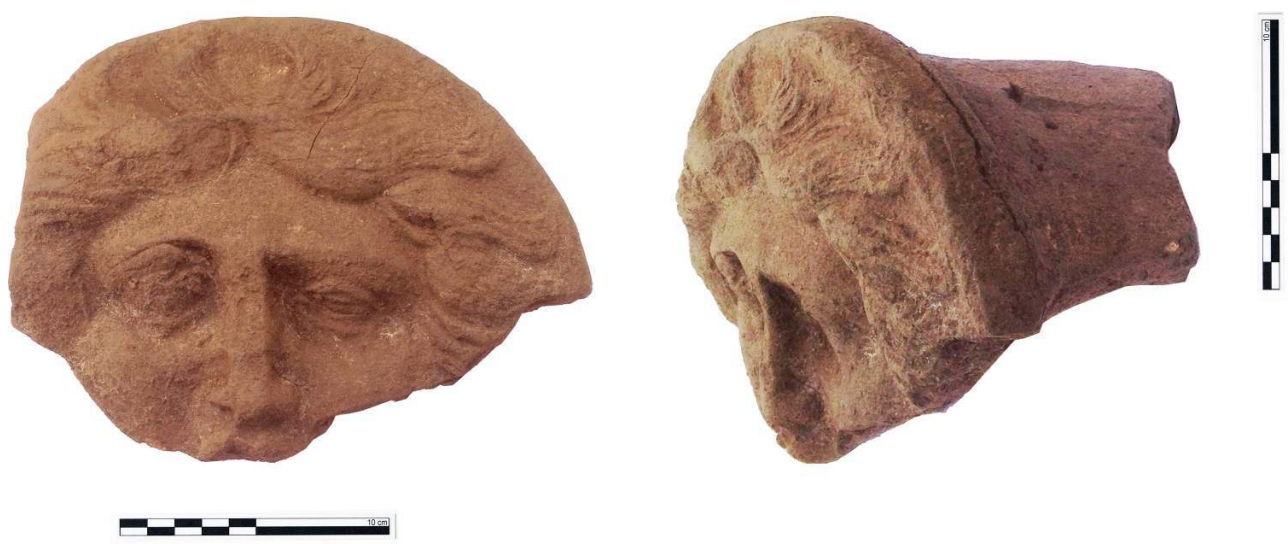

Martine Dewailly.

Nell'allargamento sud dell'area M (fig. 12), si è iniziato lo scavo dell'US $21^{15}$, dove si sono messe in luce pietre di grandi dimensioni situate ad una quota superiore rispetto al crollo esplorato nel 2011, dal quale sono separate da uno strato di terra; questo induce a pensare che il crollo si sia svolto in due fasi. Nell'angolo sud dell'area L, l'US 21 appare privo di pietre e copre, nell'angolo sud-ovest, lo strato 17 composto da pietruzze e già identificato nel 2011.

Figure 12 - Scavo dell'area $M-L$, visto verso ovest.

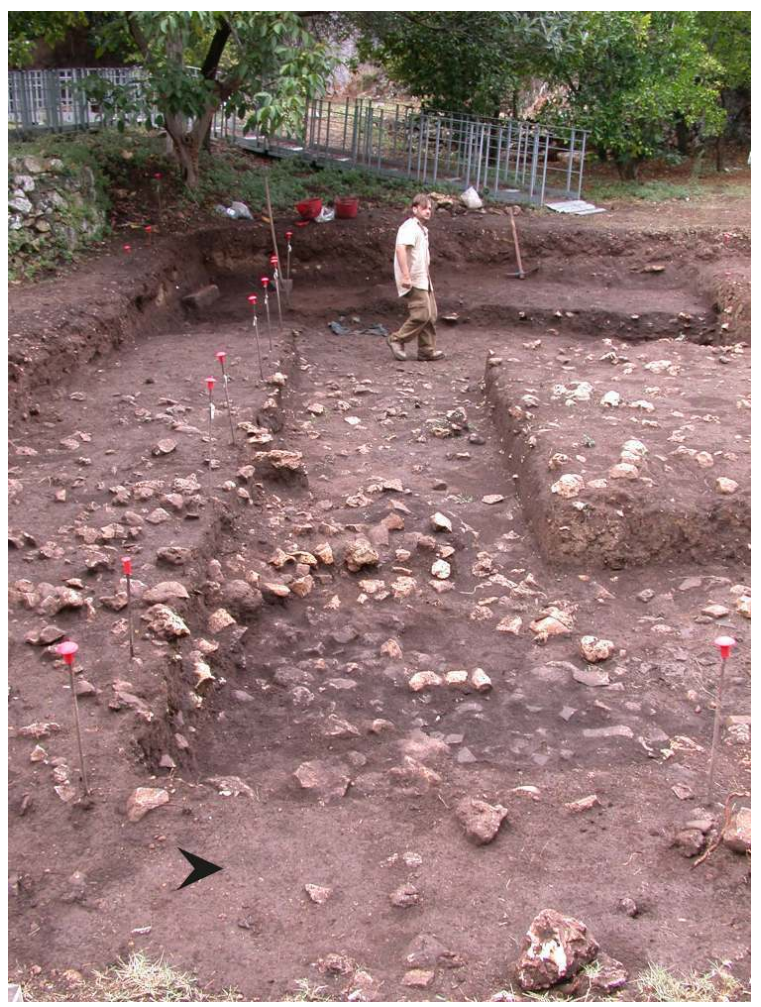

Jessica Bartolomeo.

Si è deciso pertanto di non scavarlo ma di indagarne la sua estensione negli allargamenti sud e ovest dove, effettivamente, sembra proseguire. Il materiale rinvenuto si compone 
principalmente di ceramica comune da dispensa, da fuoco e in quantità minore di ceramica da mensa, a vernice nera e di tipo Grey Ware, databili al II secolo a.C.

Nella parete sud-ovest dell'area L, è affiorato un blocco quadrangolare di grandi dimensioni che prosegue in profondità verso sud. È stato quindi deciso di lasciarlo in situ. Il blocco presenta delle modanature sui due lati a vista e un foro per l'inserimento di una grappetta, che suggeriscono la sua appartenenza ad una struttura di importanti dimensioni.

Verso nord, si ritrova l'US 16 del 2011, distinto quest'anno come US 23, e caratterizzato da una presenza maggiore di tegole rispetto alle pietre. Lo scavo ha restituito un frammento di testa femminile in terracotta ${ }^{16}$, che conserva parte del diadema che coronava la testa, della capigliatura ondulata, del fronte e dell'arco sopraccigliare sinistro (fig. 13), un elemento architettonico e alcuni frammenti bronzei, tra i quali una fibula; nel 2011, la pulizia superficiale di questo stesso strato aveva restituito una punta di giavellotto e una pinzetta da toeletta (fig. 14).

Figure 13 - Frammento di testa femminile in terracotta.
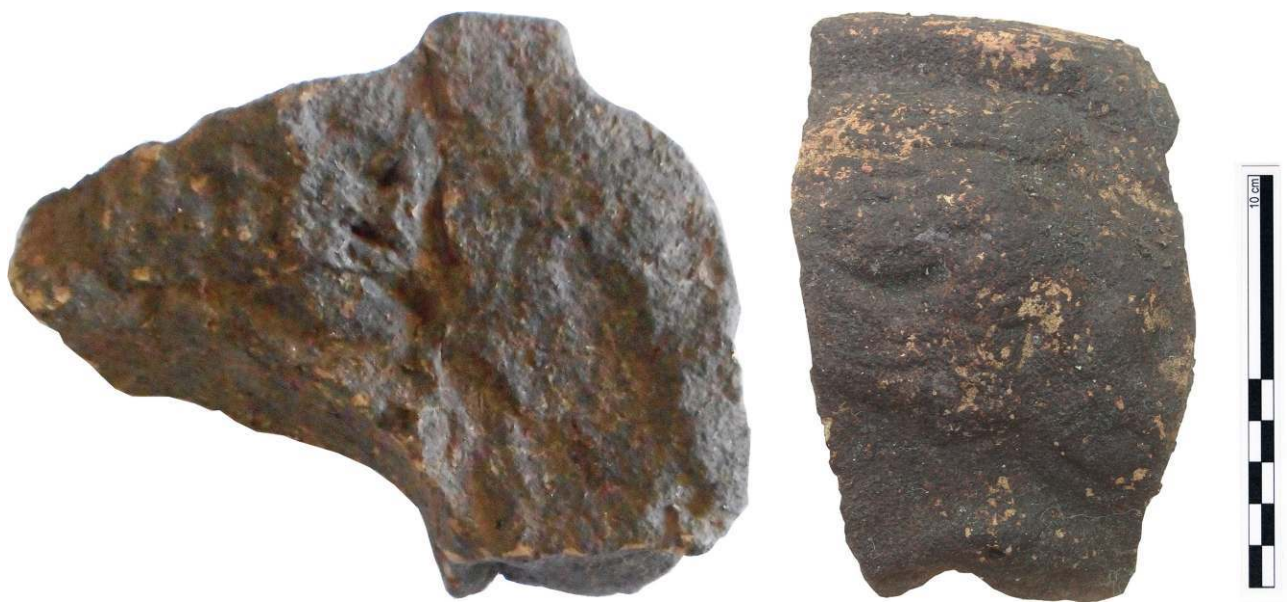

Martine Dewailly.

Le classi ceramiche sono analoghe a quelle raccolte nell'US 21, soprastante: ceramica comune da dispensa e da cucina $e$, in minore quantità, ceramica fine a vernice nera, di tipo Grey Ware e a pareti sottili. Segue lo strato, US 24, costituito di terra rossastra mista a pietruzze : esso evidenzia un pendio verso nord ed ha restituito del materiale ceramico cronologicamente coevo a quello dell'US 23.

Invece, è apparso un evidente cambio di strato, US 25, nell'angolo nord-ovest dell'area L, dove il terreno di colore giallo si presenta molto friabile. Esso corrisponderebbe allo sbriciolamento di una pietra locale, chiamata "tufina» e potrebbe essere un piano costruito di cui bisognerà indagare sia l'andamento che l'estensione. 
Figure 14 - Reperti metallici dall'area L.
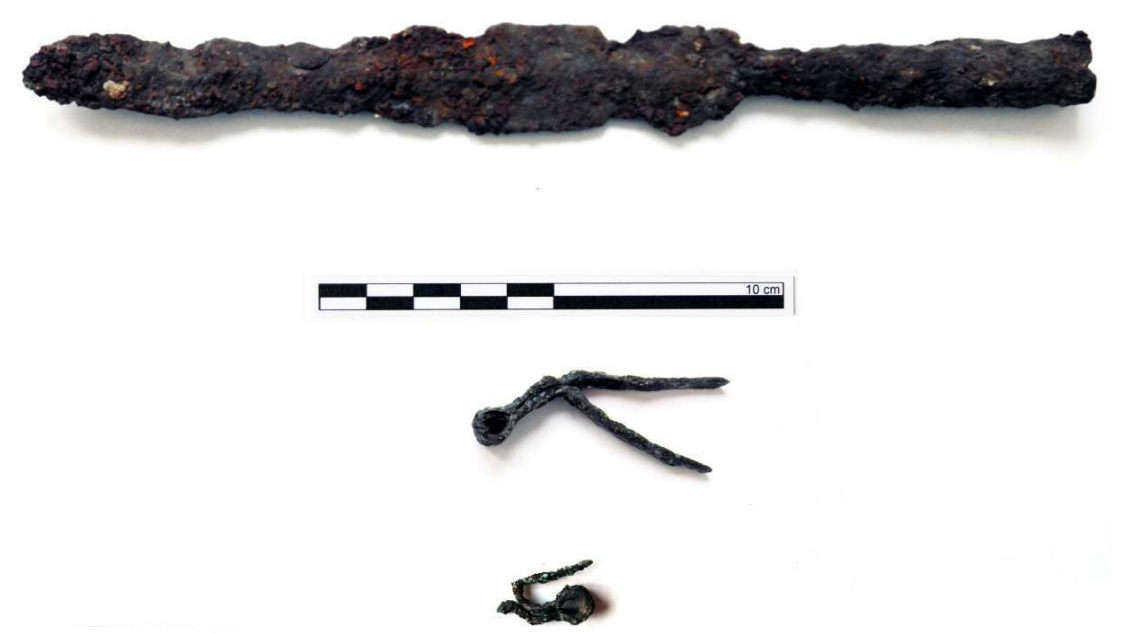

Jessica Bartolomeo, Jacopo Russo. del crollo, in particolare nell'area $M$ verso sud e verso est; lungo la parete sud, esso sembra essersi svolto in due fasi. Le dimensioni e la densità delle pietre che compongono questo crollo sono nettamente maggiori nell'area $\mathrm{M}$, dove sono presenti anche numerosi spezzoni di tegole ; questa situazione si ritrova anche nell'area L dove però le pietre e le tegole sono di piccole dimensioni. Verso nord il crollo sembra seguire un pendio piuttosto marcato.

Questi dati inducono a programmare, per le ricerche future, tre operazioni :

- l'apertura di un saggio in profondità nell'area $\mathrm{M}$, al fine di esplorare gli strati sottostanti al crollo particolarmente denso in quest'area.

- lo scavo del testimone al fine di verificare l'estensione del crollo verso nord nell'area M e L.

- il proseguimento dello scavo a nord dell'area L per verificare l'estensione del probabile livello di frequentazione.

\section{BIBLIOGRAPHIE}

Baills $2015=\mathrm{H}$. Baills, Les industries lithiques des occupations épigravettiennes de Santa Maria di Agnano (Fouilles 2007-2010), dans Bull. mus. anthropol. préhist. Monaco, 55, 2015.

Chronique 2011 = » Santa Maria di Agnano (Ostuni, Pouilles) », Chronique des activités archéologiques de l'École française de Rome [On line], URL : http://cefr.revues.org/463.

Coppola - Costantini 1983 = D. Coppola, L. Costantini, Le Néolithique ancien littoral et la diffusion des céréales dans les Pouilles durant le VIe millénaire : les sites de Fontanelle, Torre Canne et Le Macchie, in Premières communautés paysannes en Méditerranée occidentale, Montpellier, 1983.

Chronique des activités archéologiques de l'École française de Rome , Italie du Sud 
Coppola 1983 = D. Coppola, Le origini di Ostuni : testimonianze archeologiche degli avvicendamenti culturali, Martina Franca, 1983.

Coppola 2012 = D. Coppola, Il Riparo di Agnano nel Paleolitico superiore : la sepoltura Ostuni 1 ed i suoi simboli, Roma, 2012 (Università di Roma Tor Vergata).

D'Andria 1990 = F. D'Andria (éd.), Archeologia dei Messapi, Bari, 1990.

Santoro 1982 = C. Santoro, Nuovi Studi Messapici, I, Le epigrafi, Galatina, 1982.

Saturnia Tellus 2008 = D. Coppola, M. Denoyelle, M. Dewailly, I. Fusco, S. Lepetz, A. Quercia, W. Van Andringa, T. Compernolle et S. Verger, La grotte de Santa Maria di Agnano (Ostuni) et ses abords : à propos des critères d'identification d'un sanctuaire messapien, dans X. Dupré Raventos, S. Ribicchini et S. Verger (éd.), Saturnia Tellus : definizioni dello spazio consacrato in ambiente etrusco, italico, feniciopunico, iberico e celtico, Rome, 2008, p. 201-249.

\section{NOTES}

1. Coppola - Costantini 1983, in particolare p. 249-255 per Torre Canne (Gif $67256.900 \pm 80$ B.P. non calibrata).

2. Coppola 2012, p. 136.

3. Altari simili sono noti nell'area Melliche a Vaste, collocabili tra la fine del IV alla prima metà del III sec. a. C. D’Andria 1990, p. 153-157.

4. Coppola 1983, p. 123, fig. 13.

5. Santoro 1982, p. 66-67.

6. Chroniques 2011, online : http://cefr.revues.org/463. Également Baills 2015, p. 53-70, 13 fig., XII tabl.

7. Datation non calibrée.

8. MCF-Université Aix-Marseille (France) / UMR 7269 LAMPEA du CNRS.

9. Vd. Chronique 2011. Per la documentazione sugli scavi archeologici sulla terrazza superiore effettuati dal 2003 al 2011, vedere Saturnia tellus 2008.

10. Ossia gli strati corrispondenti alle US $8,9,14,15$ e 20 del 2011 ; v. nota 1.

11. Larghezza massima conservata $16,5 \mathrm{~cm}$, altezza massima conservata $13 \mathrm{~cm}$. Argilla $\mathrm{M} 2,5$ YR 8/4, con piccole inclusioni bianche.

12. L'iconografia trova un confronto con un prototipo elaborato a Taranto (Museo di Taranto, $n$ - inv. 17502) e datato tra la fine del V, inizi del IV ${ }^{\circ}$ secolo. Un'altra antefissa, rinvenuta nel terreno rimosso durante lo scavo del 2011, riproduce il viso del prototipo di Taranto. Rispetto a quest'ultimo, è di minore dimensioni e si differenzia per la presenza di un diadema.

13. L. conservata del coppo $10,5 \mathrm{~cm}, 1$. conservata $17 \mathrm{~cm}$, Alt. conservata dell'arco interno $9 / 7,5 \mathrm{~cm}$.

14. Ringraziamo V. Capozzoli (Université Paris I - Panthéon Sorbonne) per averci mandato queste prime informazioni sulle due antefisse.

15. L'US 21 equivale all'US 18 del 2011; v. nota 1.

16. Altezza massima conservata $9 \mathrm{~cm}$; larghezza massima conservata $5 \mathrm{~cm}$. Argilla M 2,5 YR 8/4, con piccole inclusioni bianche. 
INDEX

Mots-clés : sanctuaire, sépulture, occupation médiévale, influence hellénistique institutions Parc archéologique de S. Maria d'Agnano, Università degli Studi di Bari Aldo Moro, Soprintendenza per i Beni Archeologici della Puglia

\section{AUTEURS}

\section{DONATO COPPOLA}

Università degli Studi di Bari Aldo Moro - donato.coppola[at]libero.it

NICOLA DE PINTO

Università degli Studi di Bari Aldo Moro - nicola.depinto83[at]libero.it

MICHELE PELLEGRINO

Università degli Studi di Bari Aldo Moro - pellegrino.michele1990[at]gmail.com

\section{HENRY BAILLS}

UMR 7194-MNHN. Paris - hbaills[at]gmail.com

\section{MARTINE DEWAILLY}

École française de Rome - martine.dewailly[at]efrome.it

JACOPO RUSSO

Università degli studi di Roma Tor Vergata - jacorusso[at]vodafone.it

JESSICA BARTOLOMEO

Université de Neuchâtel - jessica.bartolomeo1[at]gmail.com 